\title{
La presencia del mito clásico en "To Helen" (I y II) de Edgar Allan Poe
}

\author{
Marta Mariño Mexuto \\ Universidade de Santiago de Compostela \\ mmarinom@gmail.com \\ https://dx.doi.org.10.12795/futhark.2018.il3.03
}

Fecha de recepción: 23.10 .2018

Fecha de aceptación: 0I.12.2018

Resumen: Los dos poemas de E. A. Poe titulados "To Helen", a pesar de estar dedicados a mujeres distintas, comparten una clara base clásica, en la que el ideal de mujer representado por Elena de Troya se entrecruza con otros mitos y se funde con las personas reales que inspiraron ambas obras. Al margen de las referencias míticas y geográficas que podemos encontrar, lo primordial es la idea que del mundo clásico tenía Poe y cómo la plasmaba en su obra, aspecto a menudo pasado por alto.

Palabras clave: Edgar Allan Poe, Tradición clásica, Poesía.

\section{The Classic Myth in "To Helen" (I and II) by Edgar Allan Poe}

\begin{abstract}
Both of the poems by E. A. Poe entitled To Helen, even though they were dedicated to different women, share an obvious classic base, in which the feminine archetype represented by Helen of Troy meets other myths and merges into the real people who inspired both poems. Leaving aside the mythical and geographic references which we may find, the most important element is Poe's vision of the classic world and how he expressed it in his works, an aspect which has been frequently overlooked.
\end{abstract}

Keywords: Edgar Allan Poe, Classic Tradition, Poetry.

Sumario: Introducción. I. To Helen (I). 2. To Helen (II). 3. Conclusión. 


\section{Introducción}

En $|83|$ y 1848 , es decir, al principio y al final de su carrera literaria (el autor moriría en 1849), Poe escribió dos poemas homónimos, dedicados a dos mujeres distintas, pero fuertemente relacionados entre sí. Los dos reflejan su visión de la figura mítica de Elena de Troya, pero sin perder la vinculación con la persona concreta que fue su inspiración. No son, por tanto, meros poemas de circunstancias en los que las referencias clásicas sirvan de marco o de adorno para mostrar la erudición del escritor, sino que muestran que sus conocimientos se incorporan de forma natural a la idea general del poema, como veremos a continuación.

\section{To Helen (I)}

El poema, aparecido en el volumen de $|83|^{\prime}$, estaba dedicado a Jane Stanard, la madre de uno de sus compañeros del colegio, la cual murió poco después de que Poe la conociera, lo que le causó una impresión muy profunda. El nombre Helen, con sus connotaciones clásicas, sería empleado más veces por Poe; incluso hablando de Jane Stanard con personas que no la habían conocido, se refería a ella como "Helen Stanard". El hecho de que haya dado el mismo título a un poema posterior prueba que, para Poe, Helen constituía, más que un nombre, un ideal. En palabras de Davidson,

The poem, therefore, is not about a woman, Mrs. Stanard, Mrs. Allan, nor even Helen of Troy. Its subject is the way the mind can move toward the past and, in some such symbol as the indefinable beauty of woman, is able to comprehend a world and culture long vanished from this earth. [...]. The poem is, in short, the Idea of antiquity gained through a virtual sensing of physical forms $[\ldots]^{2}$.

Las referencias clásicas se van insertando a lo largo del poema, de manera que el nombre Helen, aunque en principio no tiene por qué relacionarse con Elena de Troya, acaba por inscribirse en un marco grecolatino a medida que se suceden los versos:

\footnotetext{
' Aunque el poema apareció por primera vez en I83I, es la edición revisada de I845 la que se considera preferible y que se publica actualmente.

2 DAVIDSON, Edward H. (1976), Poe: A Critical Study, Cambridge, Harvard University Press, p. 33. 
Helen, thy beauty is to me

Like those Nicéan barks of yore,

That gently, o'er a perfumed sea,

The weary, way-worn wanderer bore

To his own native shore.

On desperate seas long wont to roam,

Thy hyacinth hair, thy classic face,

Thy Naiad airs have brought me home

To the glory that was Greece,

And the grandeur that was Rome.

Lo! in yon brilliant window-niche

How statue-like I see thee stand,

The agate lamp within thy hand!

Ah, Psyche, from the regions which

Are Holy-land!3.

La primera estrofa, en particular, tiene resonancias homéricas: la imagen de las naves nos remite, en principio, a la llíada, aunque el último verso indica que los viajeros se encuentran de vuelta a sus hogares, así que, cronológicamente, estos sucesos pertenecerían a la Odisea. En la llíada, puesto que la acción comienza cuando ya hace diez años del inicio de la guerra, no se narra la escena de las naves llegando a la costa troyana, que, sin embargo, sí aparece en otras obras, como en el primer estásimo de la Electra de Eurípides, con algunas similitudes con el poema de Poe: las naves, las divinidades marinas ${ }^{4}$, la visión idílica del mar...:

Naves ilustres que un día arrebatasteis a Troya con incontables remos escoltando la danza de las Nereidas cuando saltaba el delfín amante de la flauta ante las proas de oscuros espolones retorciéndose, acompañando al hijo de Tetis, ligero en el salto de sus pies, a Aquiles, junto con Agamenón hasta las riberas del Simoeis en Troya ${ }^{5}$.

${ }^{3}$ POE, Edgar Allan (1984): Poetry, en Poetry and Tales, ed. P. F. Quinn, Nueva York, The Library of America, p. 62.

${ }^{4}$ La náyade reaparece en otro poema de Poe: Sonnet - To Silence.

${ }^{5}$ EURÍPIDES (2004): Electra, en Tragedias II, ed. de J. L. Calvo Martínez, Madrid, Gredos, pp. 273-338, aquí 305 . 
Se trata de los mismos elementos que aparecen al comienzo del poema 64 de Catulo :

quae simul ac rostro ventosum proscidit aequor

tortaque remigio spumis incanduit unda,

emersere feri candenti e gurgite vultus

aequorae Nereides monstrum admirantes. ${ }^{7}$

Volviendo a To Helen, y siempre suponiendo que se trate de los argivos de la guerra de Troya, resulta extraño comparar la belleza de Elena con la de las naves que vuelven a Grecia tras haber derrotado a los troyanos. Desde el punto de vista geográfico, la ciudad nicena de la que provienen las naves no puede ser, como afirma David Rogers ${ }^{8}$, la Nicea que se encuentra junto al mar de Mármara, pues fue fundada por uno de los generales de Alejandro Magno, Antígono Monóftalmos en 316 a. C. $y$, por tanto, mucho después de que se escribiera la Odisea. Además, hallándose en Bitinia, formaba parte del imperio de Alejandro, pero no de Grecia, así que difícilmente puede representar el ideal de la Grecia clásica, ni temporal ni geográficamente ${ }^{9}$. Arthur Hobson Quinn también sostiene esta teoría de la Nicea bitinia, aunque con poco convencimiento:

I prefer the explanation given to me thirty years ago by my colleague, John C. Rolfe, who identified the adjective with Nicaea, a city founded by Alexander the Great on the banks of the Hydaspes, or Jelum River in India, where a fleet was built to convey a portion of the army through the Red Sea homeward. I see, however, no great light shed upon the poem by any of these interpretations of "Nicaea"10.

Si nos atenemos a la geografía y a la cronología, lo más probable es que se trate de la ciudad situada en Lócride, cerca del desfiladero de las Termópilas y con

${ }^{6}$ CATULO (20I4): Carmina, ed. de J. C. Fernández Corte y J. A. González Iglesias, Madrid, Ediciones Cátedra, p. 34I.

7 “Y, apenas escindió la veteada llanura / con su espolón, y la ola, batida por el remo, / destellaba en espumas, surgieron del luciente / torbellino unas caras salvajes: las Nereidas / marinas, admiradas de aquella maravilla" (CATULO, ibidem).

${ }^{8}$ ROGERS, David (1965): Tales and Poetry of Edgar Allan Poe, Nueva York, Monarch Press, p. 8I.

9 Aunque, evidentemente, la guerra de Troya no ocurrió en la época conocida como clásica, consideramos que se inscribe dentro de la concepción de Poe de lo clásico: la épica, las artes plásticas (en especial la escultura, con la que relaciona la belleza de Helen), la mitología... En cambio, la época helenística, por los profundos cambios que acarrea, no puede ser el escenario en el que Poe inscribe su poema.

${ }^{10}$ HOBSON QUINN, Arthur (1998): Edgar Allan Poe: A Critical Biography, Baltimore, The Johns Hopkins University Press, p. 179. 
salida al mar (existió una tercera Nicea al norte del Epiro, pero es imposible que Poe se refiriera a esa) ${ }^{11}$. Aunque no parece existir ninguna razón especial por la que quiera referirse a esta ciudad, la etimología de la palabra sería bastante clara para los lectores (gr. nikaia, "victoriosa") y así, se califica a las naves, a su vez, de victoriosas, a pesar de que se trate indiscutiblemente de un gentilicio. Además, no podemos olvidar que, muchas veces, Poe elegía los nombres por su sonoridad y lo admitía abiertamente.

Sin embargo, si olvidamos la incongruencia temporal de la Nicea bitinia, hay que tener en cuenta que esta ciudad se situaba muy cerca de Troya. Aunque el emplazamiento exacto lo descubriera Schliemann en 1868, casi veinte años después de la muerte de Poe, ya desde los siglos XVI y XVII se habían planteado diversas localizaciones que no distan mucho de la actual. Por ello, no es imposible que Poe, al llamar "nicenos" a los barcos, esté aludiendo a Troya de manera indirecta. En el poema 46 de Catulo hallamos una referencia a la llanura nicena:
lam ver egelidos refert tepores,
iam caeli furor aequinocialis
iucundis Zephyri silescit aureis.
Linquantur Phyrgii, Catulle, campi
Nicaeaeque ager uber aestuosae:
ad claras Asiae volemus urbes. ${ }^{12}$

Catulo se refiere a su viaje a Bitinia en el séquito del gobernador de esta provincia y sabemos por otros de sus poemas que, una vez allí, visitó la tumba de su hermano, cerca de la antigua Troya. Seguramente se refiera a esta región cuando habla de Nicaeaeque ager. La identificación de Bitinia con el emplazamiento de Troya se hace aún más evidente en su poema 65:

Namque mei nuper Lethaeo gurgite fratris pallidulum manans alluit unda pedem,

Troia Rhoetheo quem subter litore tellus eremptum nostris obterit ex oculis ${ }^{13}$

\footnotetext{
"Epiro era considerado por los griegos un reino bárbaro. Además, esta Nicea no tenía salida al mar.

12 "La primavera templa ya los días / otra vez, las heladas / terminan. Ya la furia / del cielo equinoccial guarda silencio / gracias al céfiro de suave soplo. / Atrás queden, Catulo, / las llanuras de Frigia, el fértil campo / niceo, demasiado caluroso. / Volemos a las célebres ciudades / de Asia [...]" (CATULO, ibidem).

13 "hace muy poco / la corriente que mana del abismo / del Leteo bañó el pálido pie / de mi hermano, cubierto ya por tierra / de Troya, junto a la retea costa / y robado a mis ojos para siempre [...] (CATULO, ibidem).
} 
A algunos críticos, como Davidson, no les preocupa mucho la ubicación de Nicea, al igual que otros términos del poema que, sin embargo, son menos enigmáticos:

Nor is it of crucial importance that we be able to attach precise interpretations to such terms as "Nicean barks", "hyacinth hair", the "agate lamp", or "Holy Land". "Helen" is quite obviously Helen of Troy $[\ldots]^{14}$

Al margen de la geografía, lo que refuerza la relación con la Odisea es también la palabra utilizada para describir al viajero, "wanderer", que indica un rumbo incierto y que, por tanto, entra en contradicción con el hecho de que vuelva a su casa ("to his own native shore"). Ulises es el wanderer por excelencia, pues en la Odisea se mezclan el deseo de regresar a su patria y las incesantes aventuras que retrasan dicho regreso. Catulo también expresa esta idea con el verbo vagare.

La historia de Troya, no obstante, es bien conocida y Poe se centra preferentemente en la descripción de Helen como el ideal de belleza y espíritu de la antigua Grecia. Describe su rostro como "classic" y dice de su cabello que es de jacinto, una comparación homérica ${ }^{15}$, como el mismo Poe nos recuerda en el relato Ligeia:

and then the raven-black, the glossy, the luxuriant and naturally-curling tresses, setting forth the full force of the Homeric epithet, "hyacinthine"! 16

La asimila también a una náyade, lo que nos evoca de nuevo el mar, aunque estas ninfas acuáticas no fueran específicamente marinas, como las nereidas. ocasiones:

Los dos últimos versos de la segunda estrofa han sido citados en multitud de

To the glory that was Greece,

And the grandeur that was Rome ${ }^{17}$.

Para Arthur Hobson Quinn, estos versos

are great ones not simply because of the alliteration, but because not two words in English could better epitomize the contrast between the civilizations of Greece and of Rome than « glory » and « grandeur ». [...] « Glory » calls up

\footnotetext{
${ }^{14}$ DAVIDSON, Edward H. (1976): op. cit., p. 32.

15 "Entonces derramó Atenea sobre su cabeza [de Ulises] abundante gracia para que pareciera más alto y más ancho e hizo que cayeran de su cabeza ensortijados cabellos semejantes a la flor del jacinto" (HOMERO [2004]: Odisea, ed. de J. L. Calvo, Madrid, Cátedra, XXIII, p. 152).

${ }^{16}$ POE, Edgar Allan (1984): op. cit., p. 263.

${ }_{17}$ POE, Edgar Allan (1984): op. cit., p. 62.
} 
the younger, brighter, more concrete culture which, through its drama and its sculpture, speaks to us with the voices of its undying art, "Grandeur » describes that more sophisticated, more abstract civilization, articulate through its laws and its power, through which we hear the tramp of the Roman legions, on their way to the conquest of the world ${ }^{18}$.

En la última estrofa, Helen es comparada físicamente con una estatua ("How statue-like I see thee stand"), lo que nos da una idea de la imagen que Poe tenía del mundo clásico, en la que las artes plásticas tenían un especial interés. Si su rostro es "classic" es precisamente porque se parece a las estatuas. El poema finaliza con una invocación a Psique que, sin duda, aparece como una personificación del alma y sin relación con la historia de Eros y Psique que se cuenta en El asno de oro de Apuleyo. Poe nombra a Psique en el mismo sentido en su poema Ulalume. Como dice Davidson, "Woman has ceased to be woman and is now "Psyche", or a titular spirit who is the connecting link with the time past" 19 .

\section{To Helen (II)}

Esta vez, el título del poema sí se corresponde con el nombre real de la mujer que lo inspiró ${ }^{20}$, Sarah Helen Whitman, con quien Poe llegó a comprometerse tras la muerte de su esposa. No obstante, antes de escribir este segundo poema en 1848, le envió el primer To Helen. Por tanto, el nombre no es tanto una alusión a la Grecia clásica como a la propia Helen Whitman. Sin embargo, Poe no podía olvidar la naturaleza de su primer poema ni el significado que le había dado a ese nombre. En una carta dirigida a ella en la que recuerda el primer encuentro entre ambos, escribe:

I saw that you were Helen -my Helen- the Helen of a thousand dreams -she whose visionary lips had so often lingered upon my own in the divine trance of passion- she whom the great Giver of all Good had prëordained to be mine mine only- if not now, alas! then at least hereafter and forever, in the Heavens ${ }^{21}$.

Es evidente que Poe se refiere aquí a Helen como un ideal, y la mujer que conoce, además de llamarse de la misma manera, se corresponde con este ideal. El poema narra también su primer encuentro en el jardín de Helen y los sentimientos que le produjo. Aunque el tema se aleja de lo clásico, no faltan las referencias:

\footnotetext{
${ }^{18}$ QUINN, Arthur Hobson (1998): op. cit., p. 178.

${ }^{19}$ DAVIDSON Edward H. (1976): op. cit., p. 34.

${ }^{20}$ Debido a su extensión, reproducimos en apéndice el texto completo del poema.

${ }^{21}$ QUINN, Arthur Hobson (1998): op. cit., p. 575.
} 
But now, at length, dear Dian sank from sight,

Into a western couch of thunder-cloud;

And thou, a ghost, amid the entombing trees

Didst glide away22.

Lo más probable es que Dian se refiera a la propia luna, que se esconde tras las nubes y deja de iluminar la escena nocturna en el jardín, de manera que Helen desaparece de la vista. La luna y la luz en general ${ }^{23}$ están presentes continuamente en el poema: al principio, la luna llena ("a full-orbed moon") introduce la descripción del jardín. Cuando Helen aparece, vestida de blanco, la luna la ilumina y, cuando ésta se esconde, todo lo demás desaparece, incluso el olor de las rosas. Sin embargo, sus ojos permanecen e iluminan y guían al mismo tiempo al poeta.

A pesar de todo esto, que nos llevaría a relacionar estrechamente a Helen con la luna, los dos últimos versos, en cambio, nos presentan sus ojos como dos Venus:

I see them still-two sweetly scintillant

Venuses, unextinguished by the sun!24

Aunque está claro que se refiere al planeta Venus, que sólo es visible al amanecer $o$ al atardecer ( $y$ que se suele conocer como lucero matutino o vespertino), llama la atención la súbita alusión a Venus, que rompe con la imagen "lunar" que Helen mantenía hasta el momento. En la obra de Poe aparece en más ocasiones la visión de Venus y Diana como dos fuerzas opuestas, relacionadas a la vez con sus atributos en la mitología clásica y con los cuerpos celestes. Donde más claramente se describe esta oposición es en el poema Ulalume, en el que la luna también tiene un papel central ${ }^{25}$. Por otra parte, Helen Whitman no pasaría por alto estas referencias, especialmente las astrológicas, ya que era una mujer excéntrica y atraída por el esoterismo ${ }^{26}$

Como ya hemos visto, el rasgo más característico de Sarah Helen Whitman eran sus ojos, que Poe califica de "divinos" ( $y$ que menciona también en sus cartas).

${ }^{22}$ POE, Edgar Allan (1984): op. cit, p. 96.

${ }^{23}$ La palabra light (empleada como sustantivo o como verbo) aparece cinco veces, además de sinónimos como lustre, glare y fire, los adjetivos bright y scintillant y los verbos illumine y enkindle.

${ }^{24}$ POE, Edgar Allan (1984): op. cit., pp. 95-97.

${ }^{25}$ El tema de la oposición entre la luna, fría, y otro cuerpo celeste, más cálido, también aparece en el poema Evening Star (POE, Edgar Allan [1984]: op. cit., p. 33), aunque en ese caso se trata de una estrella y no del planeta Venus, pues éste sólo aparece al amanecer y al anochecer y la estrella de Evening Star brilla a media noche.

${ }^{26}$ QUINN, Arthur Hobson (1998): op. cit., p. 572. 
Las divinidades cuyos ojos se evocaban más a menudo eran Atenea (normalmente se le aplica el epíteto de glaucoopis, “de ojos brillantes o grises”) y Hera (calificada de boopis, "de ojos de vaca", es decir, grandes), un hecho que Poe no podía ignorar, pero ninguna de ellas aparece en el poema. Los ojos de Helen emiten un fuego "elíseo" ("...sanctified in their elysian fire”), que debe salvar y purificar al poeta. El adjetivo hace referencia a los campos Elíseos y, por tanto, a la vida después de la muerte. Puede que Poe lo relacione con la visión cristiana del paraíso, ya que poco después añade, siempre hablando de los ojos de su amada, que se encuentran en el cielo ("and are far up in Heaven"). Sin embargo, la relación con el fuego no está muy clara.

\section{Conclusión}

La influencia clásica no es en absoluto uno de los aspectos más estudiados en la obra de Poe, y mucho menos en su poesía que, salvo los celebérrimos The Raven y Annabel Lee, no es especialmente conocida. Por eso resulta especialmente interesante ver cómo, a pesar del tiempo transcurrido entre la composición de ambos poemas y las diferentes circunstancias que los rodean, se mantiene la constante del ideal femenino representado por Elena de Troya, aunque encarnado en distintas mujeres. Por supuesto, Poe combina la base grecolatina de ambos poemas con una serie de referencias que dan como resultado una obra más compleja.

Por otro parte, es curioso señalar que, para el autor, Elena de Troya carece de todas las connotaciones negativas que el personaje ha ido acumulando a lo largo de la tradición literaria, algo sorprendente en una época en la que la figura de la mujer fatal estaba en todo su apogeo y no era ajena a la obra de Poe. Lejos de la Elena "destructora de hombres" que empuja a los pueblos a luchar entre sí, la de los poemas To Helen, aunque misteriosa, ofrece una imagen dulce y espiritual, quizá menos deudora de otras obras que de la Elena de Eurípides, quien permaneció en Egipto todos los años que duró la guerra de Troya, mientras una falsa Elena ocupaba su lugar y llevaba a griegos y troyanos a su perdición. 


\section{APÉNDICE}

\section{TO HELEN II}

I saw thee once - once only - years ago:

I must not say how many - but not many.

It was a July midnight; and from out

A full-orbed moon, that, like thine own soul, soaring,

Sought a precipitate pathway up through heaven,

There fell a silvery-silken veil of light,

With quietude, and sultriness, and slumber,

Upon the upturn'd faces of a thousand

Roses that grew in an enchanted garden,

Where no wind dared to stir, unless on tiptoe -

Fell on the upturn'd faces of those roses

That gave out, in return for the love-light,

Their odorous souls in an ecstatic death -

Fell on the upturn'd faces of these roses

That smiled and died in this parterre, enchanted

By thee, and by the poetry of thy presence.

Clad all in white, upon a violet bank

I saw thee half reclining; while the moon

Fell on the upturn'd faces of the roses,

And on thine own, upturn'd - alas, in sorrow!

Was it not Fate, that, on this July midnight -

Was it not Fate, (whose name is also Sorrow,)

That bade me pause before that garden-gate,

To breathe the incense of those slumbering roses?

No footstep stirred: the hated world all slept,

Save only thee and me. (Oh, Heaven! - oh, God!

How my heart beats in coupling those two words!)

Save only thee and me. I paused - I looked -

And in an instant all things disappeared.

(Ah, bear in mind this garden was enchanted!)

The pearly lustre of the moon went out:

The mossy banks and the meandering paths,

The happy flowers and the repining trees,

Were seen no more: the very roses' odors

Died in the arms of the adoring airs.

All - all expired save thee - save less than thou:

Save only the divine light in thine eyes - 
Save but the soul in thine uplifted eyes.

I saw but them - they were the world to me.

I saw but them - saw only them for hours -

Saw only them until the moon went down.

What wild heart-histories seemed to lie enwritten

Upon those crystalline, celestial spheres!

How dark a woe! yet how sublime a hope!

How silently serene a sea of pride!

How daring an ambition! yet how deep -

How fathomless a capacity for love!

But now, at length, dear Dian sank from sight,

Into a western couch of thunder-cloud;

And thou, a ghost, amid the entombing trees

Didst glide away. Only thine eyes remained.

They would not go - they never yet have gone;

Lighting my lonely pathway home that night,

They have not left me (as my hopes have) since.

They follow me - they lead me through the years.

They are my ministers - yet I their slave.

Their office is to illumine and enkindle -

My duty, to be saved by their bright light,

And purified in their electric fire,

And sanctified in their elysian fire.

They fill my soul with Beauty (which is Hope,)

And are far up in Heaven - the stars I kneel to

In the sad, silent watches of my night;

While even in the meridian glare of day

I see them still - two sweetly scintillant

Venuses, unextinguished by the sun! ${ }^{27}$

${ }^{27}$ POE, Edgar Allan (1984): op. cit., pp. 95-97. 
